\title{
The restriction endonuclease Tru9I is a useful tool to analyze telomere sequences separately from interstitial telomeric sequences in Arabidopsis thaliana
}

\author{
María I. Vaquero-Sedas, Miguel A. Vega-Palas \\ Instituto de Bioquímica Vegetal y Fotosíntesis, CSIC-Universidad de Sevilla, Seville, Spain \\ Email: vega-palas@ibvf.csic.es
}

Received 19 April 2012; revised 3 May 2012; accepted 24 May 2012

\begin{abstract}
We have previously described a simple method to analyze the chromatin structure of Arabidopsis telomeres independently of that of Interstitial Telomeric Sequences (ITSs). By using this method, we found that, whereas ITSs are heterochromatic, Arabidopsis telomeres exhibit euchromatic features [1]. Some concerns have been recently raised about the accuracy of this procedure [2]. Here, we summarize these concerns and justify our experimental approaches and interpretation of results.
\end{abstract}

Keywords: Arabidopsis thaliana; Telomeres; ITSs; Euchromatin; Heterochromatin

It is important to differentiate between telomeres and ITSs when the chromatin structure of telomeres is analyzed by ChIP and hybridization with a telomeric probe $[1,3]$. We have previously addressed this problem in Arabidopsis by using the frequently cutting restriction enzyme Tru9I [1,4]. Since Arabidopsis telomeres are composed of perfect telomeric repeat arrays, they remain uncut after digestion with Tru9I. In contrast, ITSs are frequently cut because they are composed of very short arrays of perfect telomeric repeats interspersed with degenerated repeats [4-7]. When Arabidopsis genomic DNA is digested with Tru9I, resolved on an agarose gel and hybridized with a telomeric probe, most of the signals corresponding to ITSs disappear. Only three ITSs bands smaller than 500 bp remain. Therefore, the signals detected above 500 bp after Tru9I digestion correspond only to telomeres. In turn, the signals detected above 500 bp when the DNA is undigested correspond to both, telomeres and ITSs [4]. This observation led us to study the chromatin structure of Arabidopsis telomeres and ITSs independently. After performing ChIP experiments, we amplified the input and the immunoprecipitated DNA samples following a whole genome amplification proto- col, resolved the DNA samples undigested or digested with Tru9I in agarose gels and hybridized them with a telomeric probe. Then, we calculated the relative enrichment of ITSs versus telomeres by comparing the signals above 500 bp. Whereas undigested signals corresponded to telomeres plus ITSs, digested signals corresponded only to telomeres [1]. By using this procedure, we found that Arabidopsis telomeres have lower levels of heterochromatic marks than ITSs (H3K9Me2, H3K27Me and DNA methylation) and higher levels of euchromatic marks (H3K4Me2, H3K9Ac and H4K16Ac) [1]. Therefore, Arabidopsis telomeres exhibit euchromatic features. Both the procedure and the results mentioned above have been recently questioned by Majerová and colleagues [2]. Following, we summarize the objections that they have raised and our comments on them:

1) We have previously reported that $70 \%$ of telomerelike hybridization signal in A. thaliana corresponds to ITSs, which are digested with frequently occurring restriction enzymes [4].

With regard to these results, Majerová and colleagues referred that: "This is, however, in a clear contradiction to the above mentioned data of Uchida et al. [6] which provide an exact content of interstitial genomic regions comprising at least 3 telomeric repeats (to exclude random hits) as $20 \%$ of the total number telomeric repeats. Thus, according to genomic data, the ratio between terminal and interstitial telomeric repeats is almost reciprocal to that deduced in [4]. This discrepancy calls the results published in [4] into question. They speculate, that when the majority of telomere probe-specific signal corresponds to ITSs, the results obtained e.g. in analysis of chromatin structure reflect rather chromatin status of ITSs than that of telomeres. However, since this assumption is controversial, as demonstrated above, further implications in that direction are not substantiated."

Majerová and colleagues suggested above [2] that most of the hybridization signals displayed after hybridizing Arabidopsis genomic DNA with a telomeric probe 
correspond to telomeres but not to ITSs. They based this suggestion in a previous in silico study by Uchida and colleagues [6], where the authors made a classification/ quantification of the Arabidopsis ITSs containing three or more perfect telomeric repeats in tandem. Majerová and colleagues assumed that these are the only ITSs that should hybridize with a telomeric probe. However, most of the ITSs present in the Arabidopsis genome contain degenerated repeats, being quite infrequent the presence of three or more tandem telomeric repeats at ITSs. For this reason, Uchida et al. did not include in their study most of the ITSs present in the Arabidopsis genome, like those present in chromosome $4[4,6]$. Nevertheless, these degenerated ITSs can also hybridize with different degrees of efficiency with a telomeric probe.

Our experimental analyses undoubtedly showed that most of the signals obtained after hybridizing Arabidopsis genomic DNA with a telomeric probe corresponded to ITSs [4]. This conclusion was supported by a micrococcal nuclease digestion analysis of the Arabidopsis genome [4]: after digesting Arabidopsis chromatin with micrococcal nuclease, resolving the resulting DNA samples in an agarose gel and hybridizing them with a telomeric probe, the nucleosome ladder displayed revealed a nucleosomal spacing similar to that of bulk chromatin [4]. If the assumption made by Majerová and colleagues were true, the nucleosomal spacing revealed by the telomeric probe should have been shorter because, in all eukaryotic systems analyzed, telomeres are known to fold into shorter nucleosomes than bulk chromatin [8].

2) We have previously reported that ITSs show heterochromatic structure while telomeres exhibit euchromatic features [1]. We analyzed histone marks and DNA methylation of ITSs and telomeres independently, using a technique based on a protocol described by Lippman et al. [9]. This protocol starts with random fragmentation of cross-linked chromatin and is followed by immunoprecipitation using an appropriate antibody. Then, the immunoprecipitated DNA is either analyzed by PCR or by hybridization. We amplified the input and the immunoprecipitated DNA samples following a whole genome amplification procedure to increase hybridization sensitivity. Then, we resolved equal amounts of the amplified DNA samples either digested with Tru9I or undigested on agarose geles and hybridized them with a telomeric probe.

With regard to this technique, Majerová and colleagues argued that: "The first problem of the technique is, of course, the amplification used in both variants of processing, which may considerably change representation of individual genome regions."

Unequal amplification of individual chromosomal loci actually occurs when whole genome amplification protocols are achieved [1]. However, this amplification bias should not affect the calculation of relative enrichment values as we did because it should happen similarly in the input and in the immunoprecipitated DNA samples. In fact, this kind of objection is not applied to genomewide ChIP-chip analyses or whole-genome expression analyses, which also involve amplification steps. In addition, some of the results that we obtained with amplified samples were confirmed without performing DNA amplifications [1].

3) Majerová and colleagues also commented that: "The other, even more serious problem with the amplification-hybridization technique lies in a fact, that an arbitrary limit of $500 \mathrm{bp}$ is chosen as a boundary between the signal corresponding to telomeres and ITSs" ... "However, the authors ignore the fact that in their approach they do not analyze integral genomic DNA, but - according to the protocol in Lippman et al. [9]—rather randomly fragmented and amplified DNA sample”... "Nevertheless, after subsequent amplification and hybridization to telomeric probe, the input DNA forms a smear starting at $500 \mathrm{bp}$. The population of these fragments thus inevitably includes (in addition to ITRs/ITSs) telomere fragments of two types: a) fragments of purely telomeric sequence (from the distal or central part of the telomere), and $b$ ) fragments generated from the proximal telomere region, which comprise, in addition to their telomere part, also an adjacent subtelomeric sequence. Consequently, the latter telomere fragments are shortened by digestion, the hybridization signal of the trimmed telomeric part is moved below the 500 bp limit, and regarded as ITSs."

In the previous paragraph, Majerová and colleagues mentioned that we attributed part of the hybridization signals corresponding to the centromeric border of telomeres to ITSs. More specifically, all these telomeric signals associated with DNA fragments shorter than 500 bp after Tru9I digestion. Although this is conceptually true, it is not really a problem in the practice because the average size of Arabidopsis telomeres is $3.75 \mathrm{~kb}$, the average distance of the first subtelomeric Tru9I sites to telomeres is about $300 \mathrm{bp}$ and the average size of the sonicated DNA samples that we obtained was about 0.75 $\mathrm{kb}$. Thus, the percentage of telomeric signals ascribed to ITSs was minimal.

4) Finally, Majerová and colleagues also argued that: "It is apparent from the results shown that even with the use of whole genome amplification the results are obtained near to the detection limit of hybridization, which raises further doubts about validity and quantitative precision of the results".

This assumption is incorrect. We obtained different expositions for all our hybridization experiments but decided not to show saturating lanes in the manuscript. We certainly had to obtain long exposures to analyze the 
results without amplifying the input and immunoprecipitated DNA samples, but still we were able to detect and quantify them [1].

In summary, we are confident that the experimental approach that we used in the past to analyze the chromatin structure of Arabidopsis telomeres is correct [1]. In consequence, the results and the conclusions that we raised from them are also correct.

\section{ACKNOWLEDGEMENTS}

This research was supported by the Spanish Ministry of Science and Innovation grant BIO2011-24794 and by FEDER funds.

\section{REFERENCES}

[1] Vaquero-Sedas, M., Gámez-Arjona, F. and Vega-Palas, M. (2010) Arabidopsis telomeres exhibit euchromatic features. Nucleic Acids Research, 39, 2007-2017. doi:10.1093/nar/gkq1119

[2] Majerová, E., Fojtová, M., Mandáková, T. and Fajkus, J. (2011) Methylation of plant telomeric DNA: What do the results say? Plant Molecular Biology, 77, 533-536. doi:10.1007/s11103-011-9834-5

[3] Vaquero-Sedas, M. and Vega-Palas, M. (2011) On the chromatin structure of eukaryotic telomeres. Epigenetics, 6, 1055-1058. doi:10.4161/epi.6.9.16845

[4] Gámez-Arjona, F., López-López, C., Vaquero-Sedas, M. and Vega-Palas, M. (2010) On the organization of the nucleosomes associated with telomeric sequences. Biochimica et Biophysica Acta, 1803, 1058-1061. doi:10.1016/j.bbamcr.2010.03.021

[5] Regad, F., Lebas, M. and Lescure, B. (1994) ITSs within the Arabidopsis thaliana genome. Journal of Molecular Biology, 239, 163-169. doi:10.1006/jmbi.1994.1360

[6] Uchida, W., Matsunaga, S., Sugiyama, R. and Kawano, S. (2002) Interstitial telomere-like repeats in the Arabidopsis thaliana genome. Genes Genetic Systems, 77, 63-67. doi:10.1266/ggs.77.63

[7] Vannier, J., Depeiges, A., White, C. and Gallego, M. (2009) ERCC1/XPF protects short telomeres from homologous recombination in Arabidopsis thaliana. PLoS Genetics, 5, e1000380. doi:10.1371/journal.pgen.1000380

[8] Pisano, S., Galati, A. and Cacchione, S. (2008) Telomeric nucleosomes: Forgotten players at chromosome ends. Cell Molecular Life Science, 65, 3553-3563. doi:10.1007

[9] Lippman, Z., Gendrel, A., Colot, V. and Martienssen, R. (2005) Profiling DNA methylation patterns using genomic tiling microarrays. Nat Methods, 2, 219-224. doi:10.1038/nmeth0305-219 